\title{
Solitary wave propagation in surface stabilized ferroelectric liquid crystal cells
}

\author{
Jang-Kun Song, M. J. Sufin, and J. K. Vij ${ }^{a}$ \\ Department of Electronic and Electrical Engineering, Trinity College, University of Dublin, Dublin 2, \\ Ireland
}

(Received 22 November 2007; accepted 16 January 2008; published online 29 February 2008)

\begin{abstract}
Solitary wave propagation in surface stabilized ferroelectric liquid crystal cells controlled by surface anchoring of the alignment layers is investigated for different conditions of alignment on the two opposite surfaces. We show that the critical field $E_{c}$, where the speed of the solitary wave becomes zero, is finite for asymmetric alignment on two surfaces. We also show that the polar anchoring energy difference $\left(\Delta w_{p}\right)$ between the alignment layers can be calculated by measuring $E_{c}$, and this depends on the rubbing condition and the material of the alignment layer. (C) 2008 American Institute of Physics. [DOI: 10.1063/1.2841670]
\end{abstract}

Nematic liquid crystal displays (LCDs) have revolutionized the display industry. However, these displays suffer from major drawbacks such as the poor image quality of fast moving objects due to slow response time and their limited viewing angle. Ferroelectric liquid crystals (FLCs), which respond by an interaction of the electric field with the spontaneous polarization $\left(P_{s}\right)$, are reported to overcome the slow response time as well as the problem of limited viewing angle. A surface stabilized FLC (SSFLC) cell, ${ }^{1,2}$ where the cell thickness is small enough to suppress the helical structure of smectic $C^{*}$, has been one of the most promising methods in display applications using FLCs. Liquid crystalline smectic $C^{*}$ phase is composed of chiral molecules that form a layered and tilted molecular structure. In SSFLC cells, usually two types of domains appear: up/down domains, where the polarization is directing upwards/ downwards, as shown in Fig. 1, or the polarization is twisted along the cell thickness direction. The boundaries of these domains move on the application of the electric field, as shown in Fig. 1. The boundary movement is a solitary wave which is a solution of a nonlinear equation with a property that energy is transmitted in a localized way. The solitary wave in a SSFLC cell is not only an interesting phenomenon scientifically but it also plays an important role in the switching property, a key factor that determines its applications. The solitary wave in a SSFLC cell was observed and studied by Handschy and Clark ${ }^{2,3}$ for the first time and was investigated in detail using computer simulations by Maclennan et al. ${ }^{4}$ and it was suggested ${ }^{5}$ that this can be used to improve the switching property in a SSFLC cell by determining the factors that control its propagation.

Recently, we reported the observation of solitary wave as an electric-field induced antiferroelectric-ferroelectric (AF-F) switching in a cell using an antiferroelectric liquid crystalline $^{6}$ (AFLC) compound. AFLC is similar to FLC except that its residual polarization is canceled out due to the oppositely aligned directors in the neighboring layers of the AF phase. Nevertheless, solitary wave in the AF-F switching is basically similar to that in a SSFLC cell because both in reality are the elastically connected liquid crystalline molecular motions that induce a transition from one to the other state or domains. However, the behavior and the mechanisms

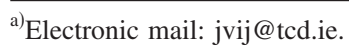

are different. While the solitary wave in the AF-F switching is governed by the interlayer interaction between the smectic layers, ${ }^{6}$ on the contrary, it is related to the surface anchoring of the alignment layers in a SSFLC cell. ${ }^{2,3}$ Hence, the nature of the surface is an essential element of the solitary wave in SSFLC cells, which has rarely been studied so far. In this letter, we characterize the solitary wave in SSFLC cells under the various surface conditions including asymmetric alignment layers. The asymmetric alignment structures with different aligning agents ${ }^{7}$ and/or different rubbing conditions $^{8}$ are designed to produce different cell structures in liquid crystal cells. Asymmetric alignment arises as an unintentional side effect caused by different surface conditions for the two cell plates such as in thin film transistor LCD (TFT LCD), where a TFT circuit is embedded on one plate and a color filter on the other plate made the surface morphology rather different.

Handschy and Clark ${ }^{2,3}$ characterized the solitary wave theoretically and determined some of its parameters in SSFLC cells including the director distortions varying along
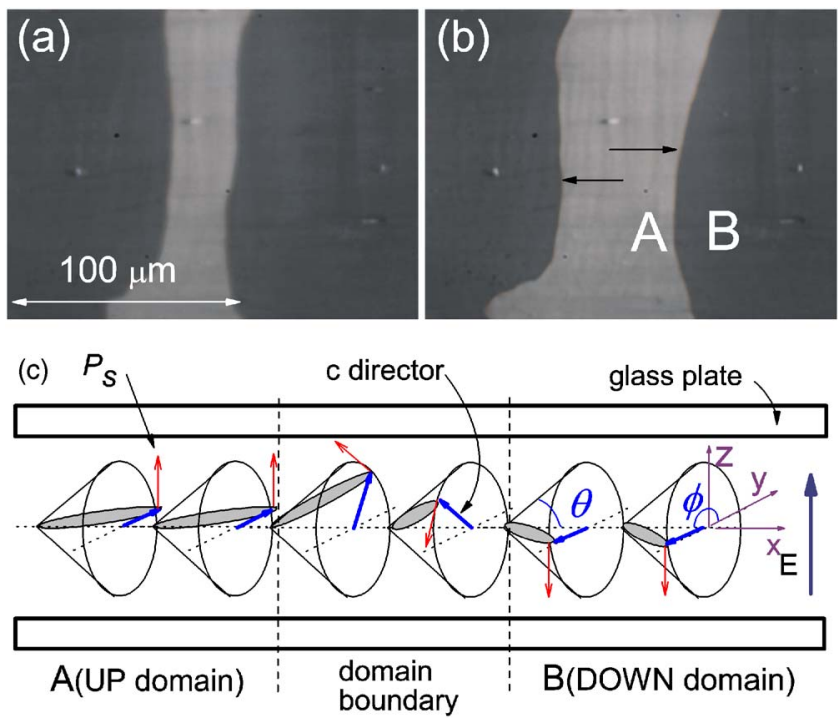

FIG. 1. (Color online) Polarizing microscopy for the solitary wave in a SSFLC; (b) the picture was taken $3 \mathrm{~s}$ after taking (a), the schematics for the solitary wave and the up/down domains and boundary (c). The directors can move on a cone, which is the necessary condition that the layer thickness does not change during switching. Smectic layers are perpendicular to the glass plates. 
the cell thickness direction and also along $X$ and $Y$ directions of window. We suggest that a simplified director distribution can easily be calculated which may allow us to investigate the additional features of the solitary wave. This is the case in the up and down domains of the SSFLC cell if a slight distortion near the surfaces is neglected, and we only consider the distortion of the director along the direction parallel to the glass plates. This simplifies the problem to be a onedimensional one. First, we consider the surface anchoring energy ${ }^{9}$ per unit surface area based on the assumption given above,

$$
\begin{aligned}
W(\phi) & =w_{0}+\left(w_{p 2}-w_{p 1}\right) \cos \phi-\left(w_{n 1}+w_{n 2}\right) \cos ^{2} \phi \\
& \equiv w_{0}+\Delta w_{p} \cos \phi-2 \bar{w}_{n} \cos ^{2} \phi,
\end{aligned}
$$

where $\phi$ is the azimuthal angle of the $\mathbf{c}$ director measured with respect to the $y$ axis. $w_{0}, w_{p}$ and $w_{n}$ are $\phi$ independent and $\phi$ dependent polar and nonpolar coefficients of anchoring energy, respectively. Note that the polar energy is proportional to $\cos \phi$ since it distinguishes the direction of polarization, while the nonpolar energy is proportional to $\cos ^{2} \phi$ since it is concerned with the relative angle of the director from the surface. The subscripts 1 and 2 represent the two surfaces. $\Delta w_{p} \equiv\left(w_{p 2}-w_{p 1}\right)$ is the polar anchoring energy difference between the two surfaces, and $\bar{w}_{n} \equiv\left(w_{n 1}+w_{n 2}\right) / 2$ is the average nonpolar anchoring energy.

A simple free energy expression can be obtained as

$$
\begin{aligned}
F & =\int\left\{K^{\prime}\left[\frac{\partial \phi(x)}{\partial x}\right]^{2}-P_{s} E \cos \phi(x)+\frac{W[\phi(x)]}{l}\right\} d V \\
& \equiv \int\left\{K^{\prime}\left[\frac{\partial \phi(x)}{\partial x}\right]^{2}+U[\phi(x)]\right\} d V,
\end{aligned}
$$

where $U[\phi(x)] \equiv w_{0} / l-\left(P_{s} E-\Delta w_{p} / l\right) \cos \phi-2 \bar{w}_{n} / l \cos ^{2} \phi$, the effective elastic coefficient $K^{\prime} \equiv K \sin ^{2} \theta / 2$, and $l$ is cell thickness. The first term in Eq. (2) is the elastic distortion energy of the solitary wave, the second term is the coupling energy of the polarization with the field, and the last term is the anchoring energy.

Corresponding to Eq. (2), the equation of motion of the solitary wave can be written as ${ }^{10-12}$

$$
\gamma \frac{\partial \phi}{\partial t}-K^{\prime} \frac{\partial^{2} \phi}{\partial x^{2}}=-\frac{d U(\phi)}{d \phi},
$$

where $\gamma$ is the viscosity of the medium.

The solution of Eq. (3) is the solitary wave and is found to be

$$
\phi(x)=2 \tan ^{-1} e^{(x-v t) / d},
$$

where

$$
\begin{aligned}
& d=(1 / 2) \sqrt{K^{\prime} l /\left(\bar{w}_{n}\right)}, \\
& v=\left[E-\Delta w_{p} /\left(l P_{s}\right)\right] P_{s} d / \gamma .
\end{aligned}
$$

Here, $d$ and $v$ represent the width and the speed of the solitary wave, respectively. Thus, the field $E_{c}$, called the critical field, where the speed of the solitary wave becomes zero, is given as

$$
E_{c}=\Delta w_{p} /\left(l P_{s}\right) .
$$

$E_{c}$ is 0 when $w_{p 1}=w_{p 2}$ but is not 0 under the asymmetric

field $E_{c}$ is not zero in these cells, and $E_{c}$ varies with the
alignment condition.
TABLE I. Surface conditions for each cell and experimentally obtained $\Delta w_{p}$. For the strong rubbing condition, the surface was rubbed four times with the same rubbing strength as for the weak process.

\begin{tabular}{lccc}
\hline \hline Acronym & Surface 1 & Surface 2 & $\Delta w_{p}\left(\mathrm{~J} / \mathrm{m}^{2}\right)$ \\
\hline SR-SR & Strong rubbing & Strong rubbing & 0 \\
SR-WR & Strong rubbing & Weak rubbing & $2.64 \times 10^{-5}$ \\
SR-NR & Strong rubbing & No rubbing & $3.80 \times 10^{-5}$ \\
SR-ITO & Strong rubbing & No alignment layer & $4.38 \times 10^{-5}$ \\
\hline \hline
\end{tabular}

The solitary wave was observed in a cell of $2 \mu \mathrm{m}$ thickness using two indium tin oxide (ITO) glass plates coated with RN-1175 (Nissan Chemical Co.) and using a FLC mixture, Felix0018 (Hoechst AG Co.). The surfaces of the alignment layer were rubbed using a commercial rubbing machine (EHC Co. Ltd., RM-50), and four types of cells, as shown in Table I, were prepared.

For the AF-F switching, it is known ${ }^{6}$ that the speed $\left(v^{\prime}\right)$ of the solitary wave corresponding to the domain movement between the two states depends on the absolute value of the applied field $|E|$; hence, $v^{\prime}(E)=v^{\prime}(-E)$. On the application of rectangular shaped ac field, the domain boundary does not oscillate but moves with a constant velocity. On the other hand, the solitary wave occurring at the boundary of the up and the down domains in a SSFLC cell depends on the sign of the applied field because the directions of $P_{s}$ in both domains are opposite to each other and $P_{s}$ favors aligning parallel to the applied field (see Fig. 1). One domain enlarges at the expense of a reduction in the size of the second domain for positive fields and vice versa. Hence, on the application of the rectangular shaped ac field, the domain boundary oscillates corresponding to the applied field. Therefore, a dc field can better be used to observe the domain movement. When a dc electric field is applied to the cell, the boundaries of the domains propagate along one direction, but the speed of solitary wave decreases with time rather quickly. This is not an intrinsic property of the solitary wave but is due to a reduction in the field across the cell caused by the ionic impurities that accumulate close to the two surfaces. This problem is solved by applying a triangular-wave form of electric signal at $10 \mathrm{~Hz}$ having a dc offset voltage. The domain boundaries oscillated with the applied field, and the offset voltage was controlled to keep the average velocity of the boundary motion to be zero. The offset field, where the average velocity of the solitary wave becomes zero, is actually $E_{c}$. This is measured just after the voltage is applied to avoid the problem due to an accumulation of ionic impurities close to the surfaces of the cell. The offset voltage increases with time as the ionic impurities accumulate. A trivial calculation shows that the velocity of the solitary wave $=$ (width of the oscillating boundary) $\times 2 f$, where $f$ is the frequency of the ac part of the applied field.

The velocity $(v)$ of the solitary wave in a usual SSFLC cell having symmetric alignment layers (SR-SR cell in Table I) was measured for different temperatures, as shown in Fig. 2. $v$ almost linearly increases with increasing field at low fields and diverges at large fields. At large fields, one state transfers to the second simultaneously in the entire area, and $v$ could, therefore, be not measured. The behavior of the solitary wave for the cells having asymmetric alignment layers, plotted in Fig. 2, is rather different. The critical electric field $E_{c}$ is not zero in these cells, and $E_{c}$ varies with the 


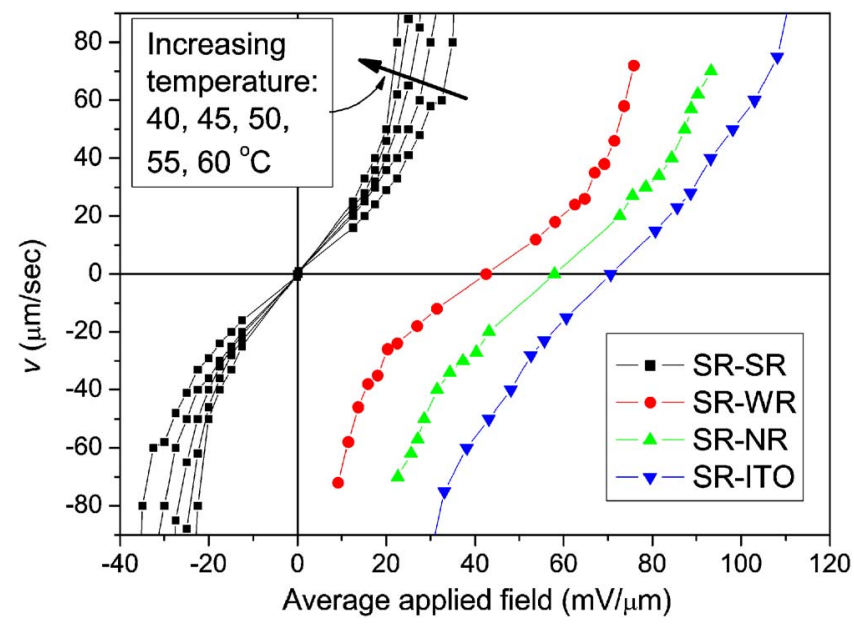

FIG. 2. (Color online) Velocity $(v)$ of the solitary wave for various conditions of the alignment layers. $v$ was measured for various temperatures for the SR-SR cell and at $40{ }^{\circ} \mathrm{C}$ for the other cells.

surface condition. This accords with Eq. (7) derived in our model. As $\Delta w_{p}$ between the two layers increases, $E_{c}$ increases, which can be confirmed by the measured values of $E_{c}$ for these cells. The values of $E_{c}$ are such that their order is $\mathrm{SR}-\mathrm{SR}<\mathrm{SR}-\mathrm{WR}<\mathrm{SR}-\mathrm{NR}<\mathrm{SR}-\mathrm{NP}$, which accords with the intuitively expected order for the differences between the two surfaces. We can calculate the polar anchoring energy difference $\Delta w_{p}$ from the experimental results by using Eq. (7); $\Delta w_{p}$ for each cell is listed in Table I.

$E_{c}$ varies considerably when measured at different points of the cells. For example, $E_{c}$ in SR-NR cell was observed varying from 70 to $110 \mathrm{mV}$ across the cell. This implies that a uniformity in rubbing is not perfect for these cells. To find the average $E_{c}$ over a wide area of the cell, we measured the optical transmittance under the cross polarizers by applying triangular wave across the cell. As shown in Fig. 3(a), $E_{s}$, which is the shift in the field of the symmetric position of the hysteresis curve from zero, is almost independent of the frequency of the applied field and shows only a slight decrease at large frequencies, as shown in Fig. 3(b). Actually, the measured $E_{s}$ was found to be almost the same as $E_{c}$ obtained by observing the solitary wave. This can easily be understood. The free energies of both domains are the same at $E_{c}$ where $v=0$. Note that the stationary boundary is obtained when the free energies of the two domains are the same. Field $E_{s}$ is actually the average transition field between the up and down domains, where the free energies of these domains should be the same. We reasonably conclude that $E_{c}=E_{s}$, and the solitary wave and the switching property are closely related to each other. We measured $E_{s}$, which is an average value over the observed area of a cell and has less error compared to $E_{c}$. The latter is obtained by observing the solitary wave. As shown in Fig. 3(c), $E_{s}$ is almost constant with temperature. This was confirmed by observing $E_{c}$ as well (data not shown). This is a rather surprising result because $E_{c}$ is inversely proportional to $P_{s}$ [Eq. (7)], the latter increases with decreasing temperature, as shown in Fig. 3(c) (open circle points). To have constant $E_{c}$ over a wide temperature range, $\Delta w_{p}$ should also be proportional to $P_{s}$. This implies that the polar anchoring energy coefficient $w_{p}$ actually depends on $P_{s}$, and the polar anchoring energy where $W_{p} \propto\left(\overrightarrow{\mathbf{P}}_{s} \cdot \hat{s}\right), \hat{s}$ is a unit vector normal to the surface of the substrate.
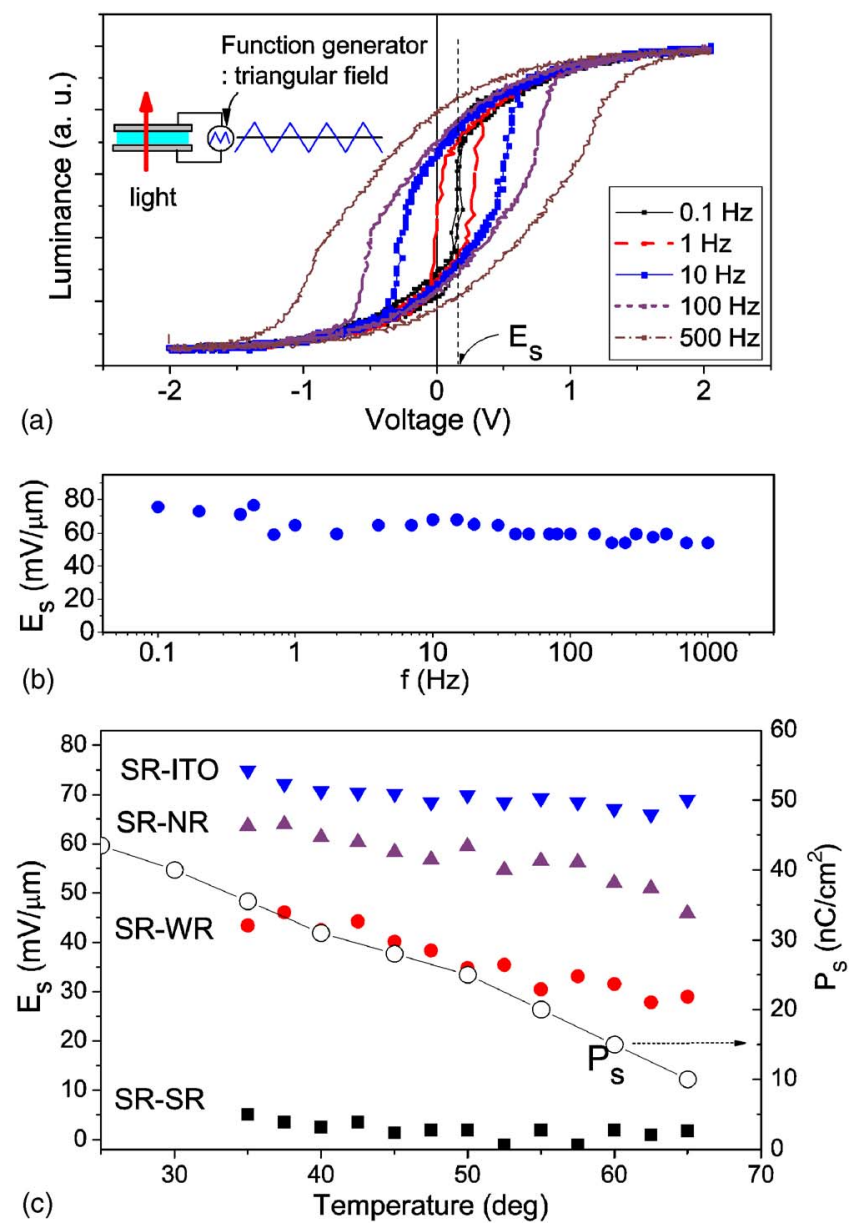

FIG. 3. (Color online) Optical transmittance vs applied field using triangular shape signal under the cross polarizers (a), where the position of the cell with the crossed polarizers is controlled to have the symmetric transmittance curve, and $E_{s}$ as a function of the frequency of the applied field (b) for SR-NR cell at $40{ }^{\circ} \mathrm{C}$. (c) shows $P_{s}$ of Felix0018 and $E_{s}$ as a function of temperature for the various cell condition at $10 \mathrm{~Hz}$.

We have modeled the solitary waves in SSFLC cells having asymmetric alignment layers and carried out the experiments which fit the model. We showed that by observing the solitary wave in SSFLC cells, the polar anchoring energy difference across the two surfaces can be obtained. We also find that the polar anchoring energy depends on $P_{s}$.

We thank the Science Foundation of Ireland (SFI) grant (No. 06/RFP/ENE039) for funding the research work and SURE program. J.K. Song thanks Samsung Electronics Co., Ltd. for granting a leave of absence from Seoul.

${ }^{1}$ N. A. Clark and S. T. Lagerwall, Appl. Phys. Lett. 36, 899 (1980).

${ }^{2}$ M. A. Handschy and N. A. Clark, Ferroelectrics 59, 69 (1984).

${ }^{3}$ M. A. Handschy and N. A. Clark, Appl. Phys. Lett. 41, 39 (1982).

${ }^{4}$ J. E. Maclennan, Q. Jiang, and N. A. Clark, Phys. Rev. E 52, 3904 (1995).

${ }^{5}$ D. C. Ulrich, M. J. Cherrill, and S. J. Elston, Liq. Cryst. 23, 797 (1997).

${ }^{6}$ J. K. Song, A. Fukuda, and J. K. Vij, Phys. Rev. E 76, 011708 (2007).

${ }^{7}$ S. A. Jewell, T. S. Taphouse, and J. R. Sambles, Appl. Phys. Lett. 87, 021106 (2005).

${ }^{8}$ S. S. Seomun, T. Fukuda, A. Fukuda, J.-G. Yoo, Yu. P. Panarin, and J. K. Vij, J. Mater. Chem. 10, 2791 (2000).

${ }^{9}$ M. A. Handschy, N. A. Clark, and S. T. Lagerwall, Phys. Rev. Lett. 51, 471 (1983).

${ }^{10}$ J. Li, X.-Y. Wang, E. Kangas, P. L. Taylor, C. Rosenblatt, Y. Suzuki, and P. E. Cladis, Phys. Rev. B 52, R13 075 (1995).

${ }^{11}$ E. Magyari, Phys. Rev. B 29, 7082 (1984).

${ }^{12}$ L. Lei, S. Changqing, S. Juelian, P. M. Lam, and H. Yun, Phys. Rev. Lett. 49, 1335 (1982). 